\title{
INDEX TO THE BIBLIOGRAPHY, SUBJECTS AND COUNTRIES
}

GENERAL ISSUES

Religions and Philoso
$109,267,483$
Social Theory and Soch
Science, 109ff., 267ff., 483
History, 115ff., 271ff., 4
Contemporary Issues, 1
275f., 489
CONTINENTS AND
COUNTRIES

Africa, 120f., 276f., $489 f$.

Congo, 277

Egypt, 121

Ghana, 490

Kenya, $121 \mathrm{f}$.

Mali, 277

Morocco, 122, 490

Nigeria, 277

Senegal, 122

South Africa, 278

America, 122f., $490 \mathrm{f}$.

Argentina, 278

Bolivia, 278
Brazil, 123, 278f., 491

Canada, 123, 279, $491 \mathrm{f}$.

Chile, 279

Cuba, 279f., 492

Dominican Republic, 492

Mexico, 280

Peru, 280 f.

Trinidad and Tobago, 281

United States of America, 124ff., 281ff., $492 \mathrm{ff}$.

Venezuela 285

Asia, 127, $285 f$.

Ceylon, 286

China, 127f., 287, $495 f$.

India, 128f., 287f., 497

Indonesia, $129 \mathrm{f}$.

Israel, 130, 498

Japan, 130, $288 \mathrm{f}$.

Korea, 289

Malaysia, 498

Singapore, 131, 498

Viet Nam, $498 \mathrm{f}$.

A ustralia and Oceania

Australia, 131
Europe, 131f., $289 \mathrm{f}$.

Austria, 132f., 290, 499

Belgium, 134f., 290, $499 f$.

Bulgaria, 500

Czechoslovakia, 291, $500 f$.

Eire, 291

Finland, 135

France, 136ff., $291 \mathrm{ff}$, , $501 \mathrm{ff}$.

Germany, 139ff., 296ff., $506 \mathrm{ff}$.

Great Britain, 146ff., 302ff., $511 \mathrm{ff}$.

Hungary, 151, 308, 515f.

Italy, 151f., 308f., 516

The Netherlands, 153f., 310, $516 f$.

Norway, 310

Poland, 517f.

Spain, 154, 311, $518 f$.

Switzerland, 154, 311f., $519 f$.

Union of Socialist Soviet Republics - Russia, 155ff., 312f., $520 \mathrm{f}$. 